\title{
Editorial
}

\section{Sir Martin Roth - A Debt of Gratitude}

The centennial anniversary of the discovery of Alzheimer's disease ends with the death of a giant who has perhaps made the greatest impact on the disease, not Alois Alzheimer, but Sir Martin Roth. Without Sir Martin's insights and experimental ingenuity, Alzheimer's disease would have remained a rare disease of the middle aged that would have to wait its turn to be understood rather than becoming the flagship leading research in neurological diseases. Roth, together with Dr. Gary Blessed and Sir Bernard Tomlinson, defined not only the high incidence of Alzheimer's disease in the aged but also developed the instruments used to measure it in life and after, the Blessed scale and the density of pathology. The legacy of this definition endures with us in the most current criteria of diagnosis and in the debate about the importance of pathology on clinical dementia. The field owes all of these men, Blessed, Tomlinson and Roth, a huge debt of gratitude and should acknowledge that without them, there would be no field.

George Perry and Mark A. Smith 